University of Nebraska - Lincoln

DigitalCommons@University of Nebraska - Lincoln

\title{
Electron-Impact Total Ionization Cross Sections of Silicon and Germanium Hydrides
}

\author{
M. A. Ali \\ Department of Chemistry, Howard University, Washington, DC \\ Y.-K. Kim \\ National Institute of Standards and Technology, Gaithersburg, Maryland \\ W. Hwang \\ National Institute of Standards and Technology, Gaithersburg, Maryland \\ N. M. Weinberger \\ National Institute of Standards and Technology, Gaithersburg, Maryland \\ M. Eugene Rudd \\ University of Nebraska - Lincoln, erudd@unl.edu
}

Follow this and additional works at: https://digitalcommons.unl.edu/physicsrudd

Part of the Physics Commons

Ali, M. A.; Kim, Y.-K.; Hwang, W.; Weinberger, N. M.; and Rudd, M. Eugene, "Electron-Impact Total Ionization Cross Sections of Silicon and Germanium Hydrides" (1997). M. Eugene Rudd Publications. 19.

https://digitalcommons.unl.edu/physicsrudd/19

This Article is brought to you for free and open access by the Research Papers in Physics and Astronomy at DigitalCommons@University of Nebraska - Lincoln. It has been accepted for inclusion in M. Eugene Rudd Publications by an authorized administrator of DigitalCommons@University of Nebraska - Lincoln. 


\title{
Electron-impact total ionization cross sections of silicon and germanium hydrides
}

\section{J. Chem. Phys. 106, 9602 (1997); DOI:10.1063/1.473842}

Issue Date: 15 June 1997

M. A. Ali

Department of Chemistry, Howard University, Washington, DC 20059

Y.-K. Kim, W. Hwang, and N. M. Weinberger

National Institute of Standards and Technology, Gaithersburg, Maryland 20899

M. E. Rudd

Department of Physics and Astronomy, University of Nebraska-Lincoln, Lincoln, Nebraska 68588-0111

\begin{abstract}
Electron-impact total ionization cross sections of some silicon and germanium compounds have been calculated by applying a new theoretical model that has been found to be reliable for a wide range of molecules. The new theory, the binary-encounter-Bethe (BEB) model, combines the binary-encounter theory and the Bethe theory for electron-impact ionization, and uses simple theoretical molecular orbital data-binding energies, average kinetic energies, and occupation numbers - which are readily available from molecular structure codes. Total ionization cross sections of $\mathrm{SiH}, \mathrm{SiH}_{2}, \mathrm{SiH}_{3}, \mathrm{SiH}_{4}, \mathrm{Si}_{2} \mathrm{H}_{6}, \mathrm{Si}\left(\mathrm{CH}_{3}\right)_{4}, \mathrm{GeH}, \mathrm{GeH}_{2}, \mathrm{GeH}_{3}, \mathrm{GeH}_{4}$, and $\mathrm{Ge}_{2} \mathrm{H}_{6}$ are presented for incident electron energies $T$ from threshold to $1 \mathrm{keV}$, and compared to available experimental data. Theory and experiment agree well for $\mathrm{SiH}_{x}, x=1-4$, from thresholds to $T<80$ $\mathrm{eV}$, while theoretical peaks occur at lower $T$ than experimental peaks for $\mathrm{SiH}_{x}, x=1-3$. No experimental data are available for germanium hydrides for comparison. The theoretical cross sections are given by a compact analytic form suitable for applications in plasma processing.

(C)1997 American Institute of Physics.
\end{abstract}

History: $\quad$ Received 27 January 1997; accepted 4 March 1997

Permalink: $\quad$ http:/link.aip.org/link/?JCPSA6/106/9602/1 


\title{
Electron-impact total ionization cross sections of silicon and germanium hydrides
}

\author{
M. A. Ali \\ Department of Chemistry, Howard University, Washington, DC 20059 \\ Y.-K. Kim, W. Hwang, ${ }^{\text {a) }}$ and N. M. Weinberger ${ }^{\text {b) }}$ \\ National Institute of Standards and Technology, Gaithersburg, Maryland 20899 \\ M. E. Rudd \\ Department of Physics and Astronomy, University of Nebraska-Lincoln, Lincoln, Nebraska 68588-0111
}

(Received 27 January 1997; accepted 4 March 1997)

\begin{abstract}
Electron-impact total ionization cross sections of some silicon and germanium compounds have been calculated by applying a new theoretical model that has been found to be reliable for a wide range of molecules. The new theory, the binary-encounter-Bethe (BEB) model, combines the binary-encounter theory and the Bethe theory for electron-impact ionization, and uses simple theoretical molecular orbital data-binding energies, average kinetic energies, and occupation numbers-which are readily available from molecular structure codes. Total ionization cross sections of $\mathrm{SiH}, \mathrm{SiH}_{2}, \mathrm{SiH}_{3}, \mathrm{SiH}_{4}, \mathrm{Si}_{2} \mathrm{H}_{6}, \mathrm{Si}\left(\mathrm{CH}_{3}\right)_{4}, \mathrm{GeH}, \mathrm{GeH}_{2}, \mathrm{GeH}_{3}, \mathrm{GeH}_{4}$, and $\mathrm{Ge}_{2} \mathrm{H}_{6}$ are presented for incident electron energies $T$ from threshold to $1 \mathrm{keV}$, and compared to available experimental data. Theory and experiment agree well for $\mathrm{SiH}_{x}, x=1-4$, from thresholds to $T$ $<80 \mathrm{eV}$, while theoretical peaks occur at lower $T$ than experimental peaks for $\mathrm{SiH}_{x}, x=1-3$. No experimental data are available for germanium hydrides for comparison. The theoretical cross sections are given by a compact analytic form suitable for applications in plasma processing. (C) 1997 American Institute of Physics. [S0021-9606(97)00922-7]
\end{abstract}

\section{INTRODUCTION}

It has been shown in previous publications that the new theoretical method, the binary-encounter-Bethe (BEB) model ${ }^{1-3}$ produces reliable electron-impact total ionization cross sections for stable molecules and radicals using as inputs simple molecular orbital data for the ground state, all of which can be obtained by using standard molecular wave function codes. There are no adjustable or fitted parameters in the theory.

The BEB model, however, does not provide details such as resonances in the continuum, vibration and/or rotational excitations concomitant with ionization, multiple ionization, and dissociative ionization. The total ionization cross section in this method is the sum of ionization cross sections for ejecting one electron from each of the occupied molecular orbitals in the ground state. The BEB cross sections were found to reproduce reliable experimental data within 5\%$15 \%$ on a large variety of molecules from the first ionization threshold to several $\mathrm{keV}$ in incident electron energies. In principle, this method can be used to calculate the total ionization cross section from any state of a molecule, provided that the state is predominantly a single configuration in character and required molecular orbital quantities are obtainable from molecular structure codes.

In the present paper, we apply the BEB model to 11 molecules of great current interest in the modeling of chemi-

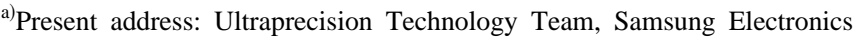
Co., Suwon, Kyonggi-do, Korea.

${ }^{b}$ Present address: Department of Chemistry, University of California, Irvine, California 92715.
}

cal vapor deposition (CVD) and plasma enhanced chemical vapor deposition (PECVD) of semiconductors: ${ }^{4-7} \mathrm{SiH}$, $\mathrm{SiH}_{2}, \mathrm{SiH}_{3}, \mathrm{SiH}_{4}, \mathrm{Si}_{2} \mathrm{H}_{6}, \mathrm{Si}\left(\mathrm{CH}_{3}\right)_{4}, \mathrm{GeH}, \mathrm{GeH}_{2}, \mathrm{GeH}_{3}$, $\mathrm{GeH}_{4}$, and $\mathrm{Ge}_{2} \mathrm{H}_{6}$. Silane $\left(\mathrm{SiH}_{4}\right)$ is widely used for plasma assisted deposition of silicon and amorphous silicon-hydride (a:SiH) films. ${ }^{8}$ Silane has also been detected on Saturn and on several other planets and satellites. As was the case for 28 molecules reported earlier, ${ }^{1-3}$ the BEB model provides cross sections in good agreement with available experimental data and sometimes enables one to discriminate between conflicting data for the same molecule. To the best of our knowledge, the total ionization cross sections for $\mathrm{GeH}_{x}, x=1-4$, and $\mathrm{Ge}_{2} \mathrm{H}_{6}$ presented here are the first of its kind in the literature.

The main advantages of the BEB model are threefold. First, the model is valid for the entire energy range from threshold to several $\mathrm{keV}$. Second, it provides compact analytic expressions for the total ionization cross section with constants characterizing occupied molecular orbitals. Third, the theory is applicable to a large variety of stable molecules and radicals as long as the ground state wave function can be calculated. The wave function need not be of very high quality.

We outline briefly the underlying theory in Sec. II, present the total ionization cross sections of individual molecules in Sec. III, and the conclusion in Sec. IV.

\section{OUTLINE OF THE THEORY}

The BEB model is a simpler version of the binaryencounter-dipole (BED) model for electron-impact ioniza- 
tion cross sections for atoms and molecules. The BED model combines the binary-encounter theory ${ }^{9}$ and the Bethe theory. ${ }^{10}$ The combination of the binary-encounter theory and the Bethe theory is achieved by requiring that the asymptotic form at high incident energy $T$ of the former, match that of the latter, both in the ionization cross section and in the stopping cross section. ${ }^{1}$ The stopping cross section is used to evaluate the stopping power of the target medium. The BED model provides a formula to calculate the singly differential cross section, or the energy distribution of ejected electrons $d \sigma / d W$, with the ejected electron energy $W$, for each atomic or molecular orbital. To apply the BED model, one needs for each occupied orbital the electron binding energy $B$, the average kinetic energy $U=\left\langle\mathbf{p}^{2} / 2 m\right\rangle$, where $\mathbf{p}$ and $m$ are the bound electron momentum and the electron mass, respectively, the electron occupation number $N$, and the continuum dipole oscillator strength $d f / d W$.

The value of the average kinetic energy $U$ for each orbital in the initial state can be obtained from any atomic or molecular wave function code that calculates the total energy in an independent particle model. To calculate $d f / d W$, however, both the initial- and continuum-state wave functions are needed, thus making the application of the BED model nontrivial. Alternatively, $d f / d W$ can be deduced from experimental photoionization cross sections. Unfortunately, such data are available for only a few stable molecules over a large energy range. Moreover, partial photoionization cross sections are needed to deduce $d f / d W$ for each orbital, and the deduction of orbital photoionization cross sections from the total is problematic ${ }^{11}$ at best.

To circumvent this difficulty, $d f / d W$ is approximated in the BEB model by choosing

$$
d f / d W=N /(B+W)^{2} .
$$

This form of $d f / d W$ closely resembles the shape of the $d f / d / W$ of the hydrogen atom in the ground state.

With this simplified $d f / d W$, the integrated ionization cross section per molecular orbital, $\sigma_{\mathrm{BEB}}$, is obtained by integrating $d \sigma / d W$ from $W=0$ to $(T-B) / 2$. The result is ${ }^{1}$

$$
\sigma_{\mathrm{BEB}}=\frac{S}{t+u+1}\left[\frac{\ln t}{2}\left(1-\frac{1}{t^{2}}\right)+1-\frac{1}{t}-\frac{\ln t}{t+1}\right],
$$

where $t=T / B, u=U / B, S=4 \pi a_{0}^{2} N R^{2} / B^{2}, a_{0}=0.5292 \AA$, and $R=13.61 \mathrm{eV}$.

In Eq. (2), the term associated with the first logarithmic function on the right-hand side (rhs) represents distant collisions (large impact parameters) dominated by the dipole interaction, and the rest of the terms on the rhs represent close collisions (small impact parameters) as described by the Mott cross section. ${ }^{12}$ The second logarithmic function originates from the interference of the direct and exchange scattering also described by the Mott cross section.

We present the values of $B, U$, and $N$ for the 11 molecules in this article in Tables I and II. The molecular orbital data were generated for the ground states of the molecules by using the molecular structure code GAMESS. ${ }^{13}$ We have omitted deep inner shell orbitals with large binding energies because they contribute little to the total ionization cross sec- tion. One can use either theoretical or experimental values of $B$ if they are available from photoionization measurements with unambiguous assignments. However, $U$ is a theoretical quantity that cannot be measured directly, although the sum of all $U$ 's is equal to the magnitude of the total energy of the target molecule, according to the virial theorem. Since experimental values of $B$ are often smaller than theoretical $B$ values, which are taken to be equal to the negative of orbital energies using the Koopman theorem, the BEB cross sections obtained using experimental $B$ values are usually higher (by $10 \%-15 \%$ at the cross section peak) than those obtained using theoretical $B$ values. Using the experimental value for the lowest electron binding energy (=first ionization potential, IP) ensures that the cross section starts at the right threshold and also leads to improved agreement of the shape and magnitude of the BEB cross section near the threshold with known experimental cross sections.

For closed-shell molecules, we used the restricted Hartree-Fock (RHF) method with the Gaussian 6-311G basis set available in the GAMESS code. For open-shell molecules, it was found ${ }^{1,2}$ that the unrestricted Hartree-Fock (UHF) method produced more realistic orbital energies for valence orbitals than the restricted open-shell Hartree-Fock (ROHF) method. We used the UHF method for open-shell molecules also with the same Gaussian 6-311G(d) basis set. Although the UHF method has the disadvantage of producing a pair of orbital and kinetic energies (associated with $\alpha$ and $\beta$ spins), the valence orbital energies from the ROHF method were often unrealistically small compared to experimental IP's, making the corresponding cross section too large. On the other hand, using separate $B$ and $U$ values for molecular orbitals associated with $\alpha$ and $\beta$ spins from the UHF method, produces BEB cross sections almost identical to those produced by using the average $B$ and $U$ values from matching $\alpha$ and $\beta$ molecular orbitals. The $B$ and $U$ values presented for open-shell molecules in Tables I and II are these average values.

In practice, the BEB model is insensitive to minor variations in the values of $B$ and $U$ used, except for the lowest $B$. Thus the use of extended basis sets other than $6-311 \mathrm{G}(\mathrm{d})$, leading to slightly altered $B$ and $U$ values, would not change the BEB cross section significantly. However, the BEB cross section is sensitive to the lowest $B$ and $U$, as the contribution to ionization cross section from the outermost occupied molecular orbital is substantial, if not dominant. For this reason, we used the experimental first IP's for the lowest $B$, which are available for many molecules, ${ }^{14}$ and theoretical values for the remaining orbitals.

\section{APPLICATION TO MOLECULES}

In this section, we compare the BEB cross sections to the experimental values of 11 molecules. As far as we are aware, no experimental cross sections are available for $\mathrm{GeH}$, $\mathrm{GeH}_{2}, \mathrm{GeH}_{3}, \mathrm{GeH}_{4}$, and $\mathrm{Ge}_{2} \mathrm{H}_{6}$. Most experiments measured the "gross" ionization cross section, i.e., the total ion current rather than the number of ions. On the other hand, BEB cross sections are "counting", ionization cross sections, 


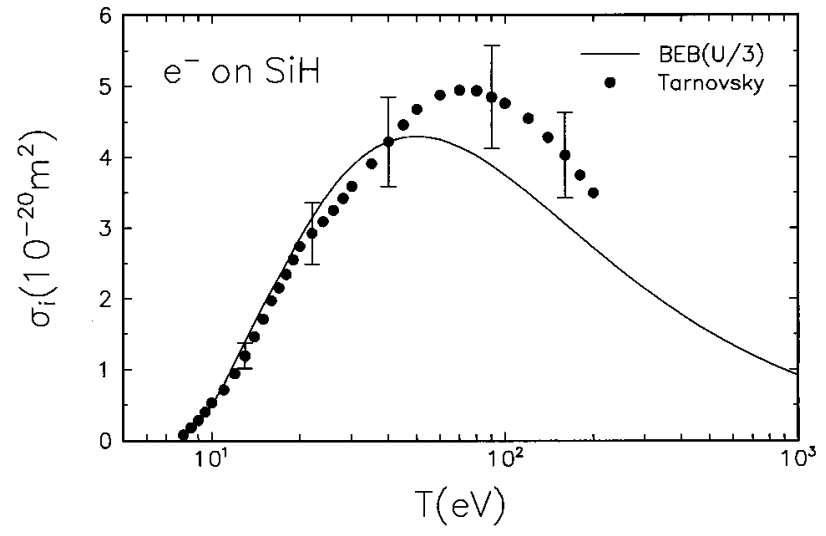

FIG. 1. Comparison of the BEB cross section to experiment for SiH. Solid curve, the present work; circles, experimental data by Tarnovsky et al. (Ref. $16)$.

which account for the number of singly charged ions produced. When a significant fraction of the ions produced are multiply charged, the gross ionization cross section will accordingly be larger than the corresponding counting ionization cross section at high $T$. Thus BEB cross sections provide lower limits to experimental gross ionization cross sections. In mass spectrometric experiments, the goal is to collect both parent molecular ions as well as all fragments at different $T$. However, since the BEB cross sections are simple sums of cross sections for ionizing one electron from each of the occupied molecular orbitals, the theory cannot provide relative cross sections for the production of different molecular fragments and ions. The separation of BEB cross sections into partial ionization cross sections for different molecular fragments and ions is not straightforward, as the crossing of different potential energy surfaces corresponding to diverse dissociative ionization and fragmentation channels must be taken into account. For instance, the BEB model cannot predict the paucity of $\mathrm{SiH}_{4}^{+}$in the ionization of $\mathrm{SiH}_{4}$ due to the preionization into $\mathrm{SiH}_{2}^{+}$observed by Berkowitz et al. ${ }^{15}$ For simplicity, we compare our theoretical cross sections to the simple sum of all experimental partial cross sections that produce an ion. Nevertheless, the comparison presented here will clearly demonstrate wide applicability of our theory and suggest that the theoretical cross sections for the other molecules not yet measured should be reliable.

\section{A. Silylidyne (SiH), Silylene $\left(\mathrm{SiH}_{2}\right)$, and Silyl $\left(\mathrm{SiH}_{3}\right)$}

For $\mathrm{SiH}$ and $\mathrm{SiH}_{3}$, we used the UHF method with the 6-311G $(d)$ basis set and we found that $\mathrm{SiH}_{3}$ has the doublet $C_{3 v}$ ground state. For $\mathrm{SiH}_{2}$, the singlet $\mathrm{C}_{2 v}$ structure is the ground state using the same basis set. The only experimental data for these molecules are by Tarnovsky et al. ${ }^{16}$ who measured absolute electron-impact ionization cross sections for $\operatorname{SiD}_{x}(x=1-3)$ from threshold to $200 \mathrm{eV}$ using a fast neutral beam technique. Since the BEB cross sections are insensitive to isotope effects, we have compared their isotopic results with the BEB cross sections with hydrogen atoms. The ions collected by Tarnovsky et al. were: $\mathrm{SiD}^{+}$and $\mathrm{Si}^{+}$for $\mathrm{SiD}$,

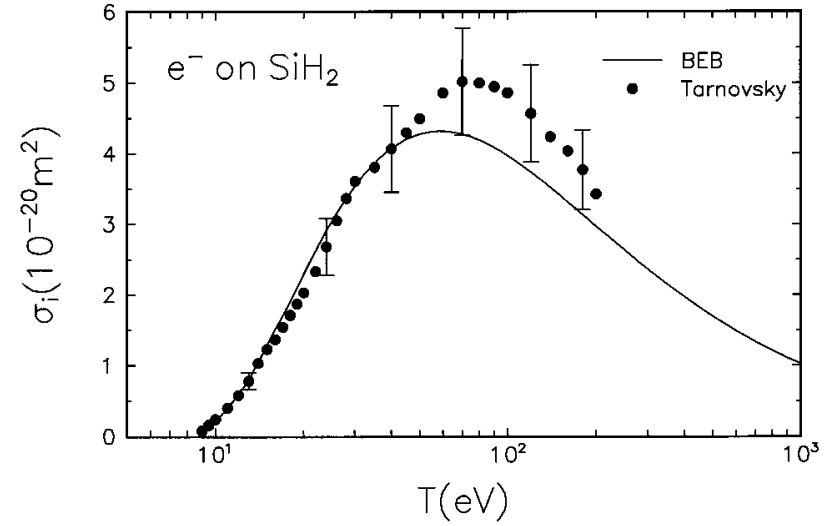

FIG. 2. Comparison of the BEB cross section to experiment for $\mathrm{SiH}_{2}$. Solid curve, the present work; circles, experimental data by Tarnovsky et al. (Ref. 16).

$\mathrm{SiD}_{2}^{+}$and $\mathrm{SiD}^{+}$for $\mathrm{SiD}_{2}$, and $\mathrm{SiD}_{3}^{+}$and $\mathrm{SiD}_{2}^{+}$for $\mathrm{SiD}_{3}$, and hence the sum of these partial cross sections should be a lower limit to the total ionization cross section. Experimental data for these molecules are compared to BEB cross sections in Figs. 1-3. Experimentally, the parent ionization cross section for all three targets $\operatorname{SiD}_{\mathrm{x}}(x=1-3)$, i.e., the production of $\mathrm{SiD}_{x}^{+}$, has essentially the same maximum value at $70 \mathrm{eV}$, and the parent ionization is the dominant process.

In a molecule with a heavy atom, the valence molecular orbitals are often dominated by an atomic valence orbital. In such a case, the $U$ value of the valence orbital tends to be high due to the radial nodes, and a high $U$ value leads to an unusually small orbital cross section. When this happens, we found it necessary to reduce the $U$ value by dividing it by the principal quantum number of the dominant atomic orbital. Since the valence molecular orbitals for the molecules in this article are the silicon $3 p$ orbital or the germanium $4 p$ orbital, we divided the appropriate $U$ values by the respective principal quantum numbers when the Mulliken population analysis indicated that the valence molecular orbitals were dominated by atomic orbitals ( $>90 \%)$. For instance, we reduced

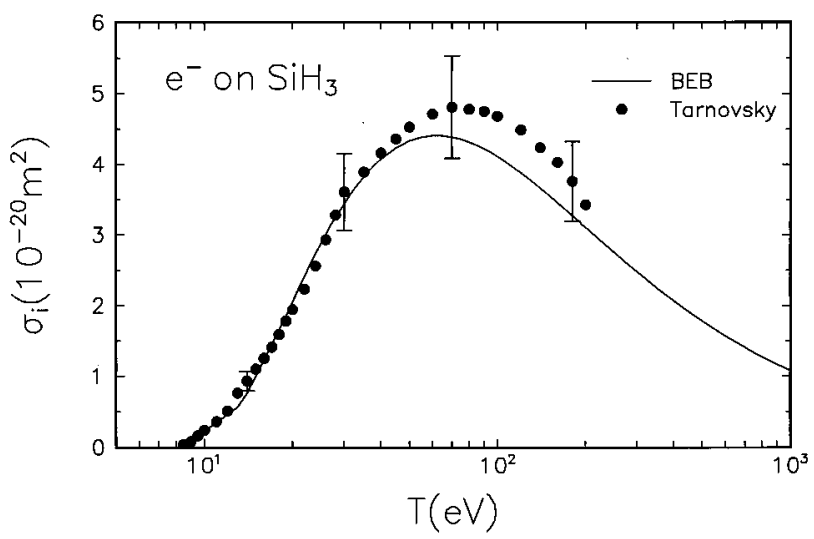

FIG. 3. Comparison of the BEB cross section to experiment for $\mathrm{SiH}_{3}$. Solid curve, the present work; circles, experimental data by Tarnovsky et al. (Ref. 16). 
the $U$ value by a factor of 3 in $\mathrm{SiH}$ and that for $\mathrm{GeH}$ by a factor of 4. The BEB cross sections calculated using the reduced $U$ values are denoted by $\operatorname{BEB}(U / 3)$ or $\operatorname{BEB}(U / 4)$, depending on the principal quantum number of the dominant atomic orbital. This increases the calculated cross section by $10 \%-15 \%$.

For germanium hydrides, some inner molecular orbitals are basically $n=3$ atomic orbitals of Ge. The $U$ values of such molecular orbitals were also divided by the principal quantum number, as indicated in Table II. This reduction increases the cross sections from inner orbitals, which in turn increases the total cross section for $T$ beyond the cross section peak by a few percent.

(a) For $\mathrm{SiH}$, the $\mathrm{BEB}(U / 3)$ cross section agrees well with the experimental results for $T<50 \mathrm{eV}$ (Fig. 1). For $T$ $>50 \mathrm{eV}$, however, the experimental values are $25 \%-$ $30 \%$ higher than the theory, reaching a maximum between 70 and $80 \mathrm{eV}$, while the BEB cross section reaches a somewhat lower maximum between 50 and $55 \mathrm{eV}$.

(b) For $\mathrm{SiH}_{2}$, again the BEB cross section agrees well with the experimental result for $T<50 \mathrm{eV}$ (Fig. 2). Similar to the case of $\mathrm{SiH}$, the experimental ionization cross section reaches a maximum between 70 and $80 \mathrm{eV}$, while the experimental values are 25\%-30\% larger than the theory for $T>60 \mathrm{eV}$. The BEB cross section has a maximum between 60 and $65 \mathrm{eV}$.

(c) For $\mathrm{SiH}_{3}$, again the experimental ionization cross section has a maximum between 70 and $80 \mathrm{eV}$, while the $\mathrm{BEB}$ cross section reaches a maximum between 55 and $60 \mathrm{eV}$ (Fig. 3).

The experimental data by Tarnovsky et al. ${ }^{16}$ for $\mathrm{SiH}$ and $\mathrm{SiH}_{3}$ show large fluctuations of the order of $20 \%-25 \%$ above $T=50 \mathrm{eV}$ in the cross sections for the production of the parent molecular ions $\left(\mathrm{SiH}^{+}\right.$and $\left.\mathrm{SiH}_{3}^{+}\right)$, which dominate the total ionization cross section. The overall experimental accuracy claimed is $\pm 15 \%$, which is indicated in Figs. 1-3. The discrepancy between theory and experiment at high $T$ may have resulted from high- $T$ events that have not been accounted for in the theory, such as multiple ionization.

\section{B. Silane $\left(\mathrm{SiH}_{4}\right)$ and Disilane $\left(\mathrm{Si}_{2} \mathrm{H}_{6}\right)$}

The experimental data for silane and disilane by Chatham et al. ${ }^{17}$ from threshold to $300 \mathrm{eV}$, and Krishnakumar and Srivastava ${ }^{18}$ from threshold to $1000 \mathrm{eV}$, are compared to BEB cross sections in Figs. 4 and 5. For $\mathrm{SiH}_{4}$, recent experimental data by Basner et al. ${ }^{19}$ agree well with the data by Chatham et al., and also with the BEB cross section calculated using the adiabatic IP of $11.65 \mathrm{eV}$, while the data by Krishnakumar and Srivastava are significantly lower than the theory at $T>30 \mathrm{eV}$. We found earlier $^{3}$ that when the adiabatic and vertical IP's are different by more than $1 \mathrm{eV}$, as is the case for $\mathrm{CH}_{4}$ and $\mathrm{NH}_{3}$, using the vertical IP for the lowest binding energy led to a much better agreement between the BEB cross section and experiment near the threshold. However, in the case of $\mathrm{SiH}_{4}$, the BEB cross sec-

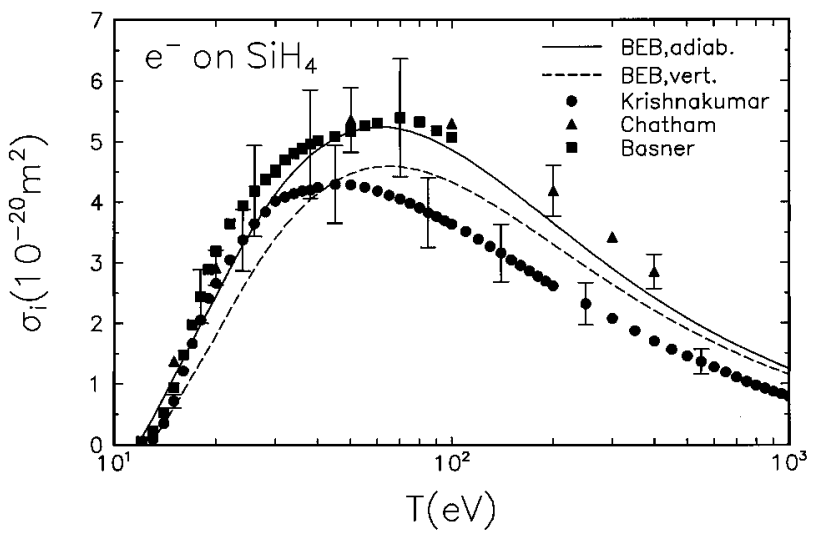

FIG. 4. Comparison of the BEB cross section to experiment for $\mathrm{SiH}_{4}$. Solid curve, the BEB cross section with adiabatic IP; dashed curve, the BEB cross section with vertical IP; circles, experimental data by Krishnakumar and Srivastava (Ref. 18); triangles, data by Chatham et al. (Ref. 17); squares, data by Basner et al. (Ref. 19).

tion with the vertical IP of $12.7 \mathrm{eV}$ deduced from the photoionization spectrum, ${ }^{20}$ is significantly smaller than all experimental data near the threshold. The BEB cross section with the vertical IP is marked "BEB, vert." in Fig. 4. We should wait until we have more examples before rendering any conclusion about the merit of using adiabatic versus vertical IP's.

For $\mathrm{Si}_{2} \mathrm{H}_{6}$, the experimental results of Chatham et al. ${ }^{17}$ and Krishnakumar and Srivastava ${ }^{18}$ differ markedly in the peak region (Fig. 5). The Chatham results resemble in general the shape of the BEB cross section but are higher in magnitude. However, if we reduce the Chatham data by a factor of 0.78, then the renormalized Chatham data agree well with BEB cross section in magnitude over the entire range of $T$. The data by Krishnakumar and Srivastava form an unusually sharp peak at $T \sim 30 \mathrm{eV}$, unlike the shape of ionization cross sections for a large number of molecules, although their data at $T>100 \mathrm{eV}$ are in excellent agreement with the BEB cross section.

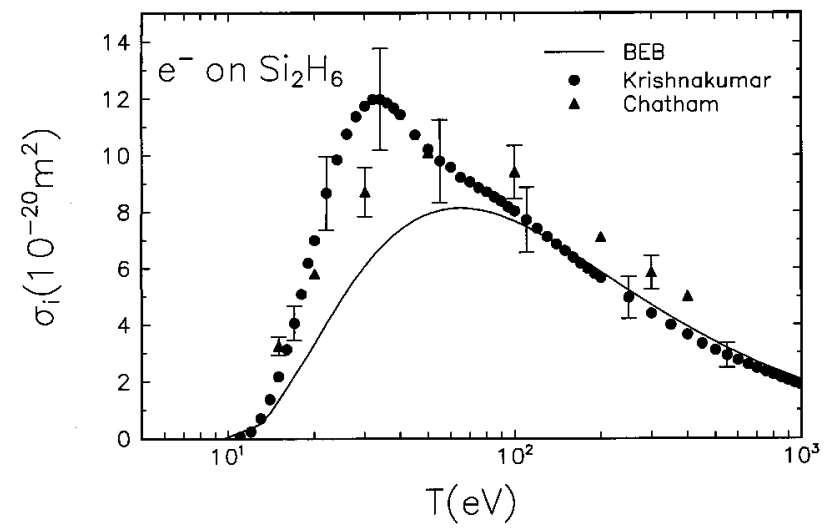

FIG. 5. Comparison of the BEB cross section to experiment for $\mathrm{Si}_{2} \mathrm{H}_{6}$. Solid curve, the present work; circles, experimental data by Krishnakumar and Srivastava (Ref. 18); triangles, data by Chatham et al. (Ref. 17). 


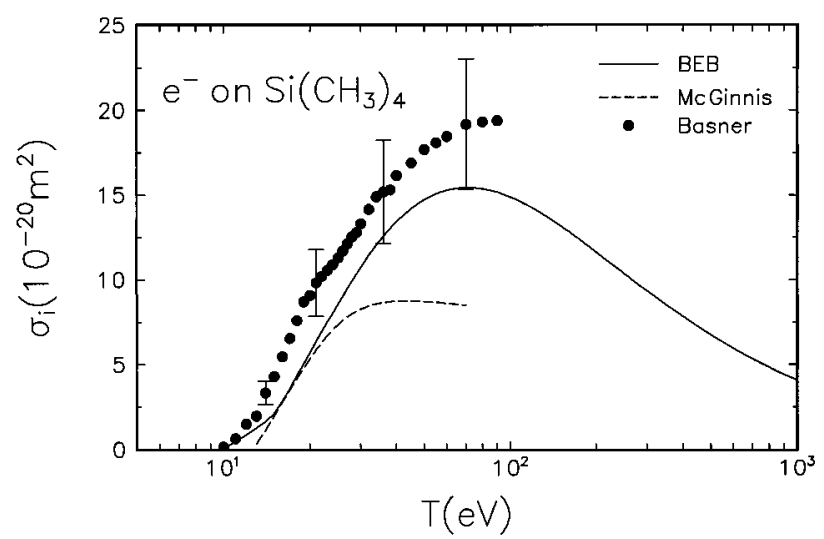

FIG. 6. Comparison of the BEB cross section to experiment for TMS, $\mathrm{Si}\left(\mathrm{CH}_{3}\right)_{4}$. Solid curve, the present work; dashed curve, experimental data by McGinnis et al. (Ref. 21); circles, data by Basner et al. (Ref. 22).

\section{Tetramethylsilane (TMS) $\mathrm{Si}\left(\mathrm{CH}_{3}\right)_{4}$}

Two sets of experimental data are available for this molecule: by McGinnis et al. ${ }^{21}$ from the threshold to $T$ $=70 \mathrm{eV}$, and by Basner et al. ${ }^{22}$ from the threshold to $T$ $=90 \mathrm{eV}$. The BEB cross section agrees in shape with the data by Basner et al., but the theory is smaller in magnitude, while the data by McGinnis et al. near the peak at $T$ $\sim 70 \mathrm{eV}$ are almost one-half of the theory (Fig. 6). The premature flattening of the data by McGinnis et al. at $T$ $>30 \mathrm{eV}$ suggests that their experiment may not have collected as many fragment ions as Basner et al. did. For instance, the combined cross section for ions of mass/charge $\leqslant 42$ collected by Basner et al., but not by McGinnis et al., at $T=70 \mathrm{eV}$ is $2.87 \times 10^{-20} \mathrm{~m}^{2}$, which will increase the peak cross section by McGinnis et al. to within $\sim 20 \%$ of the BEB cross section peak.

\section{D. $\mathrm{GeH}, \mathrm{GeH}_{2}, \mathrm{GeH}_{3}, \mathrm{GeH}_{4}, \mathrm{Ge}_{2} \mathrm{H}_{6}$}

For $\mathrm{GeH}$ and $\mathrm{GeH}_{3}$, the UHF approximation was used to obtain molecular wave functions with an internally supplied double-zeta valence (DZV) basis set with one $d$ polarization function. The binding energies and kinetic energies reported

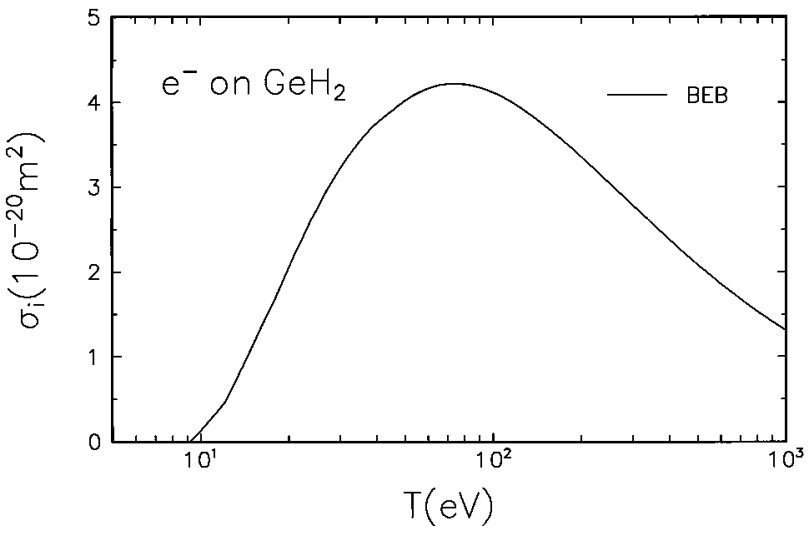

FIG. 8. The BEB cross section for $\mathrm{GeH}_{2}$.

in Table II are the average of the quantities of the corresponding $\alpha$ and $\beta$ molecular orbitals characterized by the same irreducible representation. The ground state geometry of $\mathrm{GeH}_{3}$ was found to be $C_{3 v}$ rather than $D_{3 h}$. The singlet $C_{2 v}$ state of $\mathrm{GeH}_{2}$ was found to be the ground state with the same basis set, i.e., DZV plus one $d$ polarization function in the RHF approximation. For $\mathrm{GeH}_{4}$ and $\mathrm{Ge}_{2} \mathrm{H}_{6}$, we used the DZV basis set with three $d$ polarization functions in the RHF approximation with the $T_{d}$ and $D_{2 d}$ point groups. As far as we are aware, our BEB total ionization cross sections are the only results available for these molecules. In view of the importance of these radicals and molecules in the chemical vapor deposition of $\mathrm{Ge}$, experimental verification of our results for these molecules would be worthwhile. Our BEB total ionization cross sections are shown in Figs. 7-11.

We noted earlier ${ }^{2,3}$ that the UHF approximation sometimes produces an unrealistically low binding energy for an unpaired valence electron. For instance, the theoretical binding energy for the $4 \pi$ electron of $\mathrm{GeH}$ is only $4.008 \mathrm{eV}$, which is almost certainly too low. Such a low binding energy in turn generates an unrealistically large ionization cross section. Similar low theoretical binding energy was also obtained for $\mathrm{SiH}$, but in this case an experimental IP was available. Since no experimental IP is known for $\mathrm{GeH}$, we

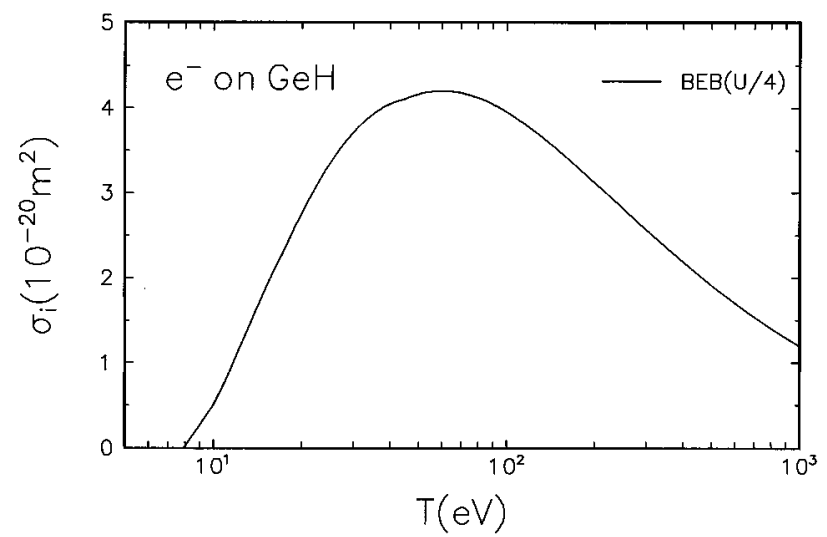

FIG. 7. The BEB cross section for $\mathrm{GeH}$.

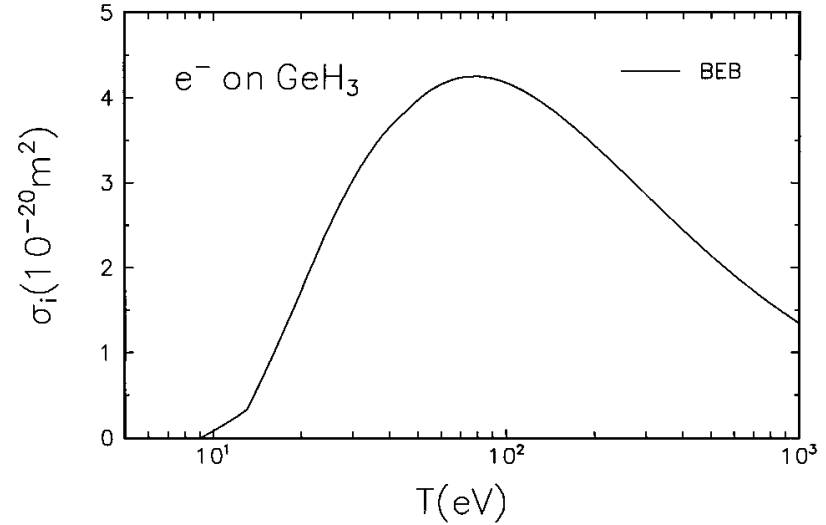

FIG. 9. The BEB cross section for $\mathrm{GeH}_{3}$. 


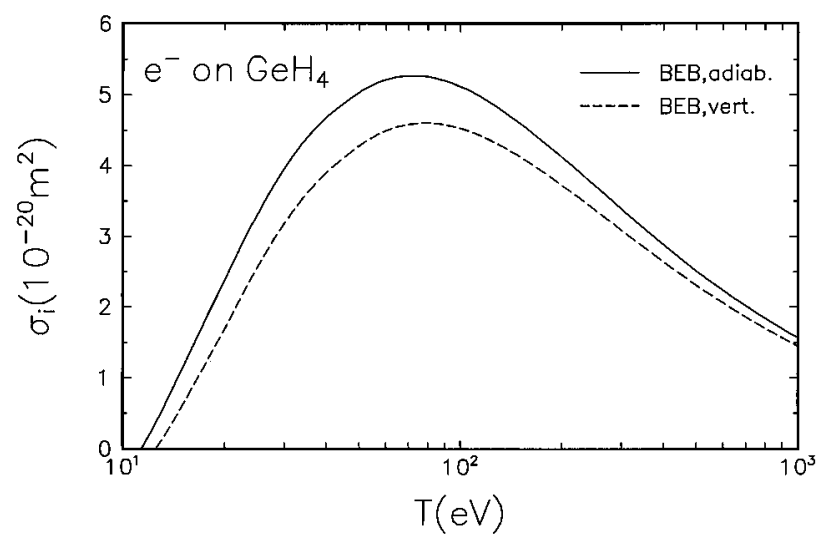

FIG. 10. The BEB cross section for $\mathrm{GeH}_{4}$. Solid curve, the BEB cross section with adiabatic IP; dashed curve, the BEB cross section with vertical IP.

obtained an "estimated" binding energy of its $4 \pi$ electron by multiplying the ratio of the experimental to theoretical binding energies of $\mathrm{SiH}$ to the theoretical binding energy of $\mathrm{GeH}$, which put the $4 \pi$ binding energy at $7.906 \mathrm{eV}$.

\section{CONCLUSION}

For the molecules where experimental ionization cross sections are available, the BEB cross sections are in good agreement with experiments at low incident energies, $T$ $<50 \mathrm{eV}$. At higher $T$, however, BEB cross sections tend to be lower than the available experimental data. This discrepancy may have come from wider fluctuations in the experimental data at higher $T$ than the general error bounds claimed by the experimenters, or some unidentified high- $T$ events, such as multiple ionization, that have not been accounted for in the theory. For unstable molecules and radicals, when only one set of measurements is available, additional independent measurements would be desirable.

When vertical and adiabatic IP's are different by more than $1 \mathrm{eV}$, such as $\mathrm{CH}_{4}$ and $\mathrm{NH}_{3}$, often as a result of the Jahn-Teller effect, we found that the use of vertical IP's significantly improved the agreement between BEB cross

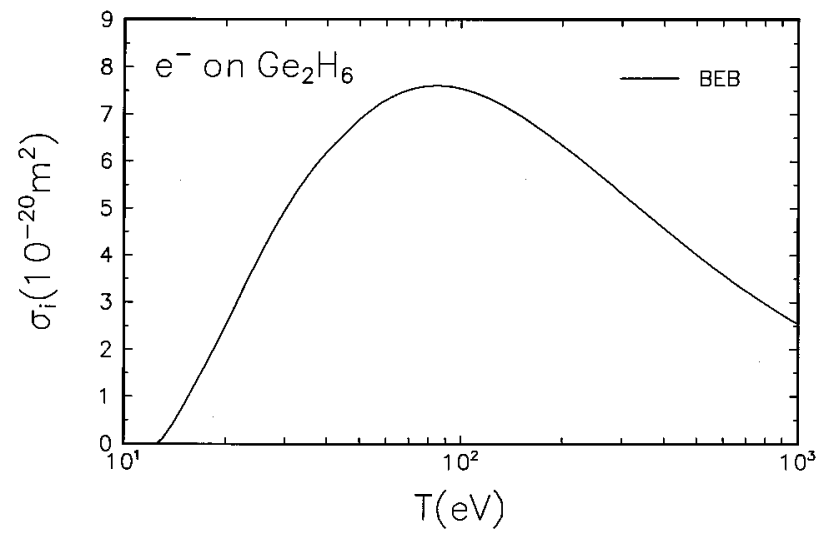

FIG. 11. The BEB cross section for $\mathrm{Ge}_{2} \mathrm{H}_{6}$.
TABLE I. Molecular orbitals, electron binding energy $B$ in eV, kinetic energy $U$ in eV, and electron occupation number $N$ for $\mathrm{SiH}, \mathrm{SiH}_{2}, \mathrm{SiH}_{3}$, $\mathrm{SiH}_{4}, \mathrm{Si}_{2} \mathrm{H}_{6}$ and $\mathrm{Si}\left(\mathrm{CH}_{3}\right)_{4}$. All $B$ and $U$ values are theoretical, except that those marked by an asterisk are experimental adiabatic IP's.

\begin{tabular}{|c|c|c|c|c|}
\hline Molecule & MO & $B$ & $U$ & $N$ \\
\hline $\mathrm{SiH}^{2} \Pi$ & $2 \sigma$ & 167.91 & 360.49 & 2 \\
\hline \multirow{6}{*}{$\begin{array}{c}\text { average of } \alpha \text { and } \beta \\
\text { orbital values }\end{array}$} & $1 \pi$ & 116.24 & 331.62 & 4 \\
\hline & $3 \sigma$ & 116.23 & 332.69 & 2 \\
\hline & $4 \sigma$ & 17.457 & 31.174 & 2 \\
\hline & $5 \sigma$ & 10.482 & 28.523 & 2 \\
\hline & $6 \sigma$ & 18.75 & 58.81 & 2 \\
\hline & $2 \pi(\mathrm{Si} 3 p)$ & $7.89^{*}$ & $21.287 / 3$ & 1 \\
\hline \multirow[t]{7}{*}{$\mathrm{SiH}_{2} C_{2 v}{ }^{1} A_{1}$} & $2 a_{1}$ & 167.24 & 360.26 & 2 \\
\hline & $1 b_{2}$ & 115.63 & 330.80 & 2 \\
\hline & $1 b_{1}$ & 115.59 & 332.16 & 2 \\
\hline & $3 a_{1}$ & 115.57 & 332.72 & 2 \\
\hline & $4 a_{1}$ & 18.414 & 29.633 & 2 \\
\hline & $2 b_{1}$ & 12.582 & 22.138 & 2 \\
\hline & $5 a_{1}$ & $8.92 *$ & 28.095 & 2 \\
\hline \multirow[t]{6}{*}{$\mathrm{SiH}_{3} C_{3 v}{ }^{2} A_{1}$} & $2 a_{1}$ & 166.88 & 359.80 & 2 \\
\hline & $3 a_{1}$ & 115.35 & 331.76 & 2 \\
\hline & $1 e$ & 115.35 & 331.92 & 4 \\
\hline & $4 a_{1}$ & 19.253 & 29.560 & 2 \\
\hline & $2 e$ & 13.304 & 22.883 & 4 \\
\hline & $5 a_{1}$ & $8.14^{*}$ & 26.787 & 1 \\
\hline \multirow[t]{4}{*}{$\mathrm{SiH}_{4} T_{d}{ }^{1} A_{1}$} & $2 a_{1}$ & 166.74 & 359.73 & 2 \\
\hline & $1 f_{2}$ & 115.20 & 331.82 & 6 \\
\hline & $3 a_{1}$ & 19.921 & 28.986 & 2 \\
\hline & $2 f_{2}$ & $11.65 *$ & 22.782 & 6 \\
\hline \multirow[t]{11}{*}{$\mathrm{Si}_{2} \mathrm{H}_{6} \quad D_{2 d}{ }^{1} A_{1}$} & $2 a_{1 g}$ & 166.71 & 359.68 & 2 \\
\hline & $2 a_{2 u}$ & 166.71 & 359.78 & 2 \\
\hline & $1 e_{u}$ & 115.19 & 331.79 & 4 \\
\hline & $3 a_{1 g}$ & 115.19 & 331.68 & 2 \\
\hline & $1 e_{g}$ & 115.19 & 331.83 & 4 \\
\hline & $3 a_{2 u}$ & 115.20 & 332.09 & 2 \\
\hline & $4 a_{1 g}$ & 20.604 & 29.637 & 2 \\
\hline & $4 a_{2 u}$ & 18.536 & 29.153 & 2 \\
\hline & $2 e_{u}$ & 13.619 & 22.212 & 4 \\
\hline & $2 e_{g}$ & 12.912 & 23.100 & 4 \\
\hline & $5 a_{1 g}$ & $9.7^{*}$ & 29.580 & 2 \\
\hline \multirow{11}{*}{$\begin{array}{c}\mathrm{Si}\left(\mathrm{CH}_{3}\right)_{4} T_{d}{ }^{1} A_{1} \\
(\mathrm{TMS})\end{array}$} & $1 f_{2}$ & 304.62 & 435.95 & 6 \\
\hline & $2 a_{1}$ & 304.62 & 435.96 & 2 \\
\hline & $3 a_{1}$ & 165.93 & 360.08 & 2 \\
\hline & $2 f_{2}$ & 114.41 & 331.91 & 6 \\
\hline & $4 a_{1}$ & 26.477 & 32.346 & 2 \\
\hline & $3 f_{2}$ & 25.070 & 33.786 & 6 \\
\hline & $5 a_{1}$ & 17.007 & 35.471 & 2 \\
\hline & $4 f_{2}$ & 15.407 & 24.504 & 6 \\
\hline & $1 e$ & 14.925 & 25.177 & 4 \\
\hline & $1 f_{1}$ & 14.376 & 26.810 & 6 \\
\hline & $5 f_{2}$ & $9.8 *$ & 34.868 & 6 \\
\hline
\end{tabular}

sections and experimental data between the threshold and the peak. ${ }^{2}$ However, we see (Fig. 4) that this is not the case for $\mathrm{SiH}_{4}$, whose vertical IP is $12.7 \mathrm{eV},{ }^{20}$ while the adiabatic IP is $11.65 \mathrm{eV}$. One feature that distinguishes $\mathrm{SiH}_{4}$ from $\mathrm{CH}_{4}$ and $\mathrm{NH}_{3}$ is that few parent ions, $\mathrm{SiH}_{4}^{+}$, are generated in the former, ${ }^{15,17}$ while the parent ions of the latter are not difficult to produce.

Intuitively, vertical IP's appear to be the right ones to use in electron-impact ionization because the ionization takes place faster than the time a molecule takes to rearrange its ionic configuration. Vertical IP's are also used in interpreting photoionization events. A comparison of the ionization cross 
TABLE II. Molecular orbitals, electron binding energy $B$ in eV, kinetic energy $U$ in $\mathrm{eV}$, and electron occupation number $N$ for $\mathrm{GeH}, \mathrm{GeH}_{2}$, $\mathrm{GeH}_{3}, \mathrm{GeH}_{4}$, and $\mathrm{Ge}_{2} \mathrm{H}_{6}$. All $B$ and $U$ values are theoretical, except that those marked by an asterisk are experimental adiabatic IP's.

\begin{tabular}{|c|c|c|c|c|}
\hline Molecule & MO & $B$ & $U$ & $N$ \\
\hline \multirow[t]{9}{*}{$\mathrm{GeH}^{2} \Pi$} & $4 \sigma$ & 193.34 & $587.95 / 3$ & 2 \\
\hline & $2 \pi$ & 141.12 & $547.90 / 3$ & 4 \\
\hline & $5 \sigma$ & 140.99 & $548.13 / 3$ & 2 \\
\hline & $1 \delta$ & 45.160 & $409.88 / 3$ & 4 \\
\hline & $3 \pi$ & 44.975 & $409.68 / 3$ & 4 \\
\hline & $6 \sigma$ & 44.975 & $408.74 / 3$ & 2 \\
\hline & $7 \sigma$ & 17.434 & 44.324 & 2 \\
\hline & $8 \sigma$ & 10.388 & 35.737 & 2 \\
\hline & $4 \pi(\mathrm{Si} 4 p)$ & 7.9068 & $26.370 / 4$ & 1 \\
\hline \multirow[t]{12}{*}{$\mathrm{GeH}_{2} C_{2 v}{ }^{1} A_{1}$} & $4 a_{1}$ & 192.80 & $587.75 / 3$ & 2 \\
\hline & $2 b_{2}$ & 140.70 & $547.69 / 3$ & 2 \\
\hline & $2 b_{1}$ & 140.52 & $548.02 / 3$ & 2 \\
\hline & $5 a_{1}$ & 140.45 & $548.18 / 3$ & 2 \\
\hline & $6 a_{1}$ & 44.744 & $409.25 / 3$ & 2 \\
\hline & $1 a_{2}$ & 44.654 & $409.66 / 3$ & 2 \\
\hline & $3 b_{2}$ & 44.567 & $409.65 / 3$ & 2 \\
\hline & $3 b_{1}$ & 44.414 & $408.14 / 3$ & 2 \\
\hline & $7 a_{1}$ & 44.308 & $409.42 / 3$ & 2 \\
\hline & $8 a_{1}$ & 18.357 & 41.292 & 2 \\
\hline & $4 b_{1}$ & 12.063 & 27.228 & 2 \\
\hline & $9 a_{1}$ & 9.132 & 35.813 & 2 \\
\hline \multirow{9}{*}{$\mathrm{GeH}_{3} C_{3 v}{ }^{2} A_{1}$} & $4 a_{1}$ & 192.73 & $587.37 / 3$ & 2 \\
\hline & $5 a_{1}$ & 140.56 & $548.09 / 3$ & 2 \\
\hline & $2 e$ & 140.55 & $548.04 / 3$ & 4 \\
\hline & $6 a_{1}$ & 44.538 & $409.09 / 3$ & 2 \\
\hline & $3 e$ & 44.578 & $408.04 / 3$ & 4 \\
\hline & $4 e$ & 44.477 & $409.30 / 3$ & 4 \\
\hline & $7 a_{1}$ & 19.343 & 40.919 & 2 \\
\hline & $5 e$ & 12.957 & 28.568 & 4 \\
\hline & $8 a_{1}$ & 9.029 & 34.153 & 1 \\
\hline \multirow[t]{6}{*}{$\mathrm{GeH}_{4} T_{d}{ }^{1} A_{1}$} & $3 a_{1}$ & 192.92 & $575.24 / 3$ & 2 \\
\hline & $2 f_{2}$ & 140.34 & $551.86 / 3$ & 6 \\
\hline & $3 f_{2}$ & 44.346 & $407.41 / 3$ & 6 \\
\hline & $1 e$ & 44.238 & $409.24 / 3$ & 4 \\
\hline & $4 a_{1}$ & 20.071 & 39.214 & 2 \\
\hline & $4 f_{2}$ & $11.33^{*}$ & 29.609 & 6 \\
\hline \multirow[t]{17}{*}{$\mathrm{Ge}_{2} \mathrm{H}_{6} \quad D_{2 d}{ }^{1} A_{1}$} & $4 a_{1 g}$ & 192.94 & $575.25 / 3$ & 2 \\
\hline & $4 a_{2 u}$ & 192.93 & $575.34 / 3$ & 2 \\
\hline & $2 e_{u}$ & 140.35 & $551.93 / 3$ & 4 \\
\hline & $2 e_{g}$ & 140.35 & $551.86 / 3$ & 4 \\
\hline & $5 a_{1 g}$ & 140.35 & $551.83 / 3$ & 2 \\
\hline & $5 a_{2 u}$ & 140.34 & $551.64 / 3$ & 2 \\
\hline & $6 a_{1 g}$ & 44.433 & $405.82 / 3$ & 2 \\
\hline & $3 e_{u}$ & 44.379 & $406.93 / 3$ & 4 \\
\hline & $3 e_{g}$ & 44.352 & $407.56 / 3$ & 4 \\
\hline & $4 e_{u}^{g}$ & 44.276 & $408.78 / 3$ & 4 \\
\hline & $4 e_{g}$ & 44.224 & $410.05 / 3$ & 4 \\
\hline & $6 a_{2 u}$ & 44.202 & $411.02 / 3$ & 2 \\
\hline & $7 a_{1 g}$ & 20.773 & $40.906 / 3$ & 2 \\
\hline & $7 a_{2 u}$ & 18.645 & 39.843 & 2 \\
\hline & $5 e_{u}$ & 13.334 & 28.632 & 4 \\
\hline & $5 e_{g}$ & 12.553 & 30.408 & 4 \\
\hline & $8 a_{1 g}$ & $12.5^{*}$ & 39.824 & 2 \\
\hline
\end{tabular}

section of $\mathrm{GeH}_{4}$ to the BEB cross section may provide further clues on the question of whether to use vertical or adiabatic IP's. For this reason, we presented two theoretical cross sections for $\mathrm{GeH}_{4}$ in Fig. 10. The BEB cross section obtained by using the adiabatic IP $(11.33 \mathrm{eV})$ is marked "BEB, adiab.," while the curve marked "BEB, vert." was obtained by using the vertical IP $(12.5 \mathrm{eV})$. Experimental vertical IP's are often not available, although adiabatic IP's are available for many molecules and radicals.

The results for germanium hydrides are new and no experimental results are available for comparison.

\section{ACKNOWLEDGMENTS}

We are grateful to the creators of GAMESS code, which was essential for this work. We thank S. K. Srivastava and $\mathrm{K}$. Becker for providing their experimental data before publication. This work at NIST was supported in part by the Office of Fusion Energy of the U.S. Department of Energy, and at the University of Nebraska-Lincoln by the National Science Foundation Grant No. PHY-9119818. One of us (M.A.A.) gratefully acknowledges the use of NIST facilities for this work as a guest scientist.

${ }^{1}$ Y.-K. Kim and M. E. Rudd, Phys. Rev. A 50, 3954 (1994).

${ }^{2}$ W. Hwang, Y.-K. Kim, and M. E. Rudd, J. Chem. Phys. 104, 2956 (1996).

${ }^{3}$ Y.-K. Kim, W. Hwang, N. M. Weinberger, M. A. Ali, and M. E. Rudd, J. Chem. Phys. 106, 1026 (1997).

${ }^{4}$ J. M. Jasinski, B. S. Meyerson, and B. A. Scott, Ann. Rev. Phys. Chem. 87, 109 (1987).

${ }^{5}$ T. Glenewinkel-Meyer, J. A. Bartz, G. M. Thorson, and F. F. Crim, J. Chem. Phys. 99, 5944 (1993), and references therein.

${ }^{6}$ P. Fons, T. Motooka, K. Awazu, and H. Onuki, Appl. Surf. Science 79/80, 476 (1994).

${ }^{7}$ G. Turban, Pure Appl. Chem. 56, 215 (1984).

${ }^{8}$ D. J. Meyer and T. I. Kamins, Thin Solid Films 222, 30 (1992).

${ }^{9}$ L. Vriens, in Case Studies in Atomic Physics, edited by E. W. McDaniel and M. R. C. McDowell (North Holland, Amsterdam, 1969), Vol. 1, p. 335 .

${ }^{10}$ H. Bethe, Ann. Physik 5, 325 (1930)

${ }^{11}$ J. Berkowitz, Photoabsorption, Photoionization and Photoelectron Spectroscopy (Academic, New York, 1979).

${ }^{12}$ N. F. Mott, Proc. R. Soc. Lond. Ser. A 126, 259 (1930).

${ }^{13}$ M. W. Schmidt, K. K. Baldridge, J. A. Boatz, S. T. Elbert, M. S. Gordon, J. H. Jensen, S. Koseki, N. Matsunaga, K. A. Nguyen, S. J. Su, T. L. Windus, M. Dupuis, and J. A. Montgomery, J. Comput. Chem. 14, 1347 (1993)

${ }^{14}$ S. G. Lias, J. F. Liebman, R. D. Levin, and S. A. Kafafi, "NIST Positive Ion Energetics Database, Version 2.0," Standard Reference Database 19A, National Institute of Standards and Technology, Oct. 1993. This database lists adiabatic IP's.

${ }^{15}$ J. Berkowitz, J. P. Greene, and H. Cho, J. Chem. Phys. 86, 1235 (1987).

${ }^{16}$ V. Tarnovsky, H. Deutsch, and K. Becker, J. Chem. Phys. 105, 6315 (1996).

${ }^{17}$ H. Chatham, D. Hills, R. Robertson, and A. Gallagher, J. Chem. Phys. 81, 1770 (1984).

${ }^{18}$ E. Krishnakumar and S. K. Srivastava, Contrib. Plasma Phys. 35, 395 (1995).

${ }^{19}$ R. Basner, M. Schmidt, V. Tarnovsky, and K. Becker, J. Chem. Phys. (in press).

${ }^{20}$ A. W. Potts and W. C. Price, Proc. R. Soc. Lond. Ser. A 326, 165 (1972). A weighted average of appropriate photoionization spectra was used to determine the vertical IP's.

${ }^{21}$ S. McGinnis, K. Riehl, and P. D. Haaland, Chem. Phys. Lett. 232, 99 (1995).

${ }^{22}$ R. Basner, R. Foest, M. Schmidt, F. Sigeneger, P. Kurunczi, K. Becker, and H. Deutsch, Int. J. Mass. Spectrom. Ion Processes 153, 65 (1996). 